\title{
Completely Positive Stochastic Linear Maps Over AFD-Factors and Unitary Mixing on Generating U.H.F.E.Subalgebras
}

By

\author{
Peter M. Alberti*
}

\begin{abstract}
Provided $\mathfrak{M}$ is a non-finite AFD-factor, it it shown that the action of a completely positive, state-pre-serving linear map (stochastic map) on normal states over $\mathfrak{M}$ can always be reduced to a unitary mixing mapping with respect to a generating U.H.F.-subalgebra.
\end{abstract}

\section{$\S$ 耳. The Problem}

Let $\mathfrak{M}$ be a vN-algebra acting over some separable infinite-dimensional Hilbert-space $\mathfrak{S}$, with dual $\mathfrak{M}^{*}$ and predual $\mathfrak{M E}_{*}$, and set of normal states $S_{0}(\mathfrak{M})$. A linear map $\Phi: \mathfrak{M}^{*} \rightarrow \mathfrak{M}^{*}$ is referred to as being c.p.-stochastic over $\mathfrak{M}^{*}$, and the set of all these mappings is denoted by $S T\left(\mathfrak{M}^{*}\right)$, if $\Phi$ maps states into states, and the adjoint $\Phi^{\#}$ being a completely positive, unital linear transformation over $\mathfrak{M}^{* *}$ (the second dual of $\mathfrak{M}$ ). Analogously, a linear map $\psi: \mathfrak{M}_{*} \rightarrow \mathfrak{M N}_{*}$ is said to be c.p.-stochastic over $\mathfrak{M E}_{*}$, and the set of all these maps be $S T\left(\mathfrak{M E}_{*}\right)$, if the adjoint $\psi^{*}$ is completely positive and unital over $\mathfrak{M}$. Note that in the latter case $\psi$ throws normal states into normal ones, and $\psi^{\#}$ is also a normal positive linear mapping in the usual sense. Let us denote by $C P_{1}(\mathfrak{M})$ and $C P_{1}^{0}(\mathfrak{M})$, respectively, the sets of unital c.p.-linear mappings over $\mathfrak{M}$ and normal unital c.p. linear maps over Me, respectively.

The main goal of this paper is to establish necessary and sufficient conditions one has to impose on two n-tuples (n-a fixed but arbitrary natural number) $\{\omega\}=\left\{\omega_{1}, \cdots, \omega_{n}\right\} \subset S_{0}(\mathfrak{M}),\{\sigma\}=\left\{\sigma_{1}, \cdots, \sigma_{n}\right\} \subset S_{0}(\mathfrak{M})$ of normal states such that there is $\Phi \in S T\left(\mathfrak{M}^{*}\right)$ with $\omega_{k}=\Phi\left(\sigma_{k}\right)$ for all $k=1,2, \cdots, n$. Call this problem the

Communicated by H. Araki, February 24, 1986.

* Sektion Marhematik und Naturwissenschaftlich-Theoretisches Zentrum (NTZ), Karl-MarxUniversität Leipzig, DDR-7010 Leipzig. 
n-tuple-problem (for normal states on the $\mathrm{vN}$-algebra $\mathfrak{M}$ ).

\section{§2. Remark on the Problem}

We have in mind further applications of this $n$-tople-problem in Mathematical Physics, especially Statistical Physics. The classes of vN-algebras occuring there are rather special ones. Therefore, we restrict considerations to AFD-algebras from the very beginning, and within this category to factors. Due to the work of Connes [1] several algebraic characterizations for AFDfactors exist, all relating to the structure of complete positivity. Especially, the characterization of AFD-factors in [1] allows one to make use of the powerful property of semidiscreteness of Effros and Lance [2] in the form proved by Choi and Effros [3]. When this is seen in context with [4] and a recent useful technical result of Haagerup [5] on the form of c.p.-maps over generating U.H.F.subalgebras, we will be led to the fact that the conditions in question are equivalent to a unitary mixing problem with respect to some $C^{*}$-substructure, provided $\mathfrak{M}$ is properly infinite. In sect 9 it will be indicated how to arrive at a solution of the $n$-tuple-problem in more general cases (non-factorial situations and the remaining cases of finite AFD-factors and the finite dimensional factors). This solution will be heavily based on the non-finite AFD-factor case which is our main concern in this paper. In very special situations solutions of the $n$ tuple-problem exist, cf. the remark in 9.

\section{§3. The Main Result}

In order to formulate the result, let us consider $\mathfrak{M}$ to be a properly infinite AFD-factor acting over $\mathfrak{C}$. Assume $\mathfrak{M}_{1} \subset \mathfrak{M}_{2} \subset \mathfrak{M}_{3} \subset \ldots \subset \mathfrak{M}$ is an increasing sequence of finite-dimensional subfactors of $\mathfrak{M}\left(1\right.$ be common to all $\mathfrak{M}_{k}$ ).

Let $\mathfrak{N}_{0}=\cup \mathfrak{M}_{k}$ be the union of all these subfactors, and suppose the uniform closure $\mathfrak{A}$ of $\mathfrak{U}_{0}$ generates the $v N$-algebra $\mathfrak{M}$, i.e. $\mathfrak{U}^{\prime \prime}=\mathfrak{M}$. Then, $\mathfrak{U}$ will be referred to as a generating U.H.F.-subalgebra of $\mathfrak{M}$.

Let $\mathcal{U}(\mathfrak{U})$ denote the unitary group of $\mathfrak{A}$, and $\mathfrak{B}\left(\mathfrak{H}^{*}\right)$ be the set of all bounded linear operators over the dual $\mathfrak{A} *$ of $\mathfrak{A}$ into $\mathfrak{2} *$. For $u \in \mathfrak{A}$ let us define $T_{u} \in \mathfrak{B}\left(\mathfrak{2}{ }^{*}\right)$ through the setting $\left(T_{u} \mu\right)(x)=\mu\left(u^{*} x u\right)$, for all $x \in \mathfrak{U}$, where $\mu \in \mathfrak{Q}{ }^{*}$. We put $\mathcal{C}_{u}(\mathfrak{U})=$ point- $w^{*}$-closure of conv $\left\{T_{v}: v \in \mathfrak{U}(\mathfrak{U})\right\}$, where the point- $w^{*}$ topology is defined through the system $p_{\nu, a}(T)=|(T \nu)(a)|, \nu \in \mathfrak{U}^{*}, a \in \mathfrak{U}$, of semi-norms over $T \in \mathfrak{B}\left(\mathfrak{2}{ }^{*}\right)$. Then, $\mathcal{C}_{u}(\mathfrak{U})$ is a convex compact subset of $\mathfrak{B}(\mathfrak{2} *)$ with respect to the topology mentioned, by well-known facts. We may now 
formulate the main result.

Theorem. Given normal states $\omega_{1}, \cdots, \omega_{n}$ and $\sigma_{1}, \cdots, \sigma_{n} \in S_{0}(\mathrm{MN})$ over the properly infinite $A F D$-factor $\mathfrak{M}$ the following conditions are mutually equivalent:

$$
\begin{aligned}
& \exists \Phi \in S T(\mathfrak{M} *) \text { with } \omega_{k}=\Phi\left(\sigma_{k}\right) \text { for } \forall k: \\
& \exists \Theta \in \mathcal{C}_{u}(\mathfrak{A}) \text { such that } \omega_{k / 2 t}=\Theta\left(\sigma_{k / \mathfrak{V}}\right) \text { for } \forall k, \\
& \text { (u'ith } \left.\nu_{/ 2 \mathbb{X}} \text { indicating the restriction of } \nu \text { onto } \mathfrak{A}\right) \text {. }
\end{aligned}
$$

With other words, the action of an element of $S T\left(\mathfrak{M}^{*}\right)$ over normal states into normal states can be reduced or replaced by (convex) unitary mixing with respect to any generating U.H.F. $C^{*}$-subalgebra $\mathfrak{2}$.

Another useful form of the result is the Corollary given in the last section of this paper, which adds the result of Lemma 1 to the assertion above (cf. Section 5).

\section{\$4. Remarliss on the $\mathbb{R}$ esull and Turther Applications}

The bounded linear operators $\mathfrak{B}(\mathfrak{S})$ over the separable Hilbert-space $\mathfrak{S}$ give the standard example of a properly infinite type $\mathbb{I}$ AFD-factor. In this special case the result has been derived in [8] without making use of the "injectivity machinery" of [1]-[5]. Besides the fact that the result is of interest in its own rights, in [8] implications are given for applications of the assertion of the Theorem. Especially, the case of tuples (i.e. $n=2$ ) is of interest. In this case, the special result on $\mathfrak{B}(\mathfrak{S})$ has been used to propose and to discuss a reasonable structure for functionals which generalize the notion of "transition probability" to cases of mixed (normal) states. On the other hand, it has been shown that the proposed set of functionals is a sufficient system in the sense of a modified theory of majorization (for c.p.-stochastic maps over operator algebras). Due to the theorem above applications in the sense mentioned will be possible also in cases of other algebras than $\mathfrak{B}(\mathfrak{E})$. Those applications will be discussed in detail in a forthcoming paper. Concerning references and some recent results in this direction cf. [8], [9]. Relating the n-tuple-problem over other $W^{*}$-algebras, the commutative case has been represented in [6], [7] in detail (cf. also references quoted there), and the atomic type case has been considered in [10]. But note that even in the known cases of the n-tupleproblem mentioned, by Lemma 1 of this paper an important new piece of information will be provided (see the Corollary of the last section). 


\section{§5. Some Technical Results on a General vN-Algebra}

In this section $\mathfrak{M}$ might be an arbitrary vN-algebra. If $\{\nu\} \subset S_{0}(\mathfrak{M})$ ) and $\{x\} \subset \mathfrak{M}_{+}$are $n$-tuples of normal states and positive elements of $\mathfrak{M}$, respectively, let us define

$$
K_{n}(\{\nu\},\{x\})=\sup _{T} \sum_{k} \nu_{k} \circ T\left(x_{k}\right),
$$

where the supremum extends over all $T \in C P_{1}^{0}(\mathfrak{M})$, i.e. runs over the normal, unital completely positive linear maps.

Lemma 1. For n-tuples $\{\omega\},\{\sigma\} \subset S_{0}(\mathfrak{M})$ the following conditions are equivalent to each other:

$$
\begin{aligned}
& \omega_{k}=\Phi\left(\sigma_{k}\right), \forall k, \text { with } \Phi \in S T\left(\mathfrak{M}^{*}\right) ; \\
& \omega_{k}=\Phi\left(\sigma_{k}\right), \forall k, \text { with } \Phi \in S T\left(\mathfrak{M}_{*}\right) ; \\
& \omega_{k}=\sigma_{k} \circ T, \forall k, \text { with } T \in C P_{1}(\mathfrak{M}) ; \\
& \omega_{k}=\sigma_{k} \circ T, \forall k, \text { with } T \in C P_{1}^{0}(\mathfrak{M}) ; \\
& K_{n}(\{\omega\},\{x\}) \leq K_{n}(\{\sigma\},\{x\}) \text { for all }\{x\} \subset \mathfrak{M}_{+} .
\end{aligned}
$$

Proof. Since the sequence of implications $(2) \Rightarrow(4) \Rightarrow(3) \Rightarrow(1)$ is obvious, we will be done with the equivalences among the conditions (1)-(4) if the implication $(1) \Rightarrow(2)$ can be shown. We are going to do this. By $[11 ; 1.17 .7]$ there exists a uniquely determined central projection $z$ in $\mathfrak{M}^{* *}$ such that $R_{z} \nu=\nu\left(z\left({ }^{\circ}\right)\right)$ for every $\nu \in \mathfrak{M}^{*}$ is orthogonal to the whole $\mathfrak{M}_{*}$, and the positive linear mapping $R_{1-z}$ sends elements of $\mathfrak{M R}^{*}$ into elements of $\mathfrak{M}_{*}: R_{1-z} \mathfrak{M}^{*}=\mathfrak{M}_{*}$, with $R_{1-z} \circ R_{1-z}=R_{1-z}$. Let $\nu \in S_{0}(\mathfrak{M})$ be arbitrarily chosen. Define $\Omega \in S T\left(\mathfrak{M}^{*}\right)$ by $\Omega(\omega)=R_{1-z} \omega+\left(R_{z} \omega\right)(1) \nu, \omega \in \mathfrak{M} *$. The map $\Omega$ is positive, maps states into states and has range in $\mathfrak{M}_{*}$, obviously. $\Omega$ is also completely positive, for it is the restriction onto $\mathfrak{M}^{*} \simeq\left(\mathfrak{M}^{* *}\right)_{*}$ of the adjoint $R^{*}$ of the positive linear map $R: x \mapsto z^{\perp} x z^{\perp}+\nu(x) z$ over $\mathfrak{M}^{* *}$, i.e. $\Omega=R^{\sharp} / \mathfrak{M}^{*}$, and $R$ is completely positive by construction. Therefore, $\Omega$ is c.p.-stochastic with range in $\mathfrak{M}_{*}$. This makes it being useful in our context: define $\psi=\Omega \circ \Phi$, with the $\Phi$ of (1), and due to the properties of $\Omega$ and since all states involved are normal, we will find that $\psi\left(\sigma_{k}\right)$ $=\Omega\left(\omega_{k}\right)=\omega_{k}, \forall k$, and $\psi\left(\mathfrak{M}^{*}\right) \subset \mathfrak{M}_{*}$. Therefore, the restriction $\psi / \mathfrak{M}_{*}$ of $\psi$ onto the predual maps $\{\sigma\}$ into $\{\omega\}$ as required and is contained in $S T\left(\mathfrak{M}_{*}\right)$ by the discussion above. Therefore, we might take this map for the $\Phi$ in (2), i.e. (1) $\Rightarrow$ (2) is shown.

To see that (5) is equivalent with each of (1)-(4), by the first part of the proof the verification of $(3) \Leftrightarrow(5)$ will be sufficient. For this sake, let us define a set of 
n-tuples

$$
\mathcal{C}\{\sigma\}=\left\{\{\nu\} \subset S_{0}(\mathfrak{M}): \exists T \in \mathbb{C P}_{1}(\mathfrak{M} \mathcal{Q}) \text { with } \nu_{k}=\sigma_{k} \circ T, \forall k\right\}
$$

$\mathcal{C}\{\sigma\}$ is a convex set, obviously $\left(C P_{1}(\mathfrak{M})\right.$ is convex). We show that $\mathcal{C}\{\sigma\}$ is uniformly closed. Let $\{\nu\} \in$ norm-closure of $\mathcal{C}\{\sigma\}$. Then, since $S_{0}(\mathfrak{M} \mathcal{E})$ is uniformly closed (since $\mathfrak{M}_{*}$ is complete with respect to the functional norm) $\{\nu\} \subset S_{0}(\mathfrak{M})$ necessarily. Assume $\left\{T^{(n)}\right\} \subset C P_{1}(\mathfrak{M})$ such that $\nu_{k}^{(n)}=\sigma_{k} \cdot T^{(n)}$ and norm-lim $\nu_{k}^{(n)}$ $=\nu_{k}$, for all $k$. By point- $\sigma$-weak compactness of $C P_{1}(\mathfrak{D})$, there is a subnet $\left\{T^{\left(n_{\lambda}\right)}\right\}$ such that point- $\sigma_{w}-\lim T^{\left(n_{\lambda}\right)}=T$, for some $T \in C P_{1}\left(\mathfrak{M}_{\mathcal{C}}\right)$. Therefore, for all $x \in \mathfrak{M} \mathcal{M}: \nu_{k}(x)=\lim \nu_{k}^{\left(n_{\lambda}\right)}(x)=\lim \sigma_{k} \circ T^{\left(n_{\lambda}\right)}(x)=\sigma_{k} \circ T(x)$, for any $k$. This proves $\{\nu\} \in \mathcal{C}\{\sigma\}$. Hence $\mathcal{C}\{\sigma\}$ is closed. Together these facts imply convexity and closedness of $\mathcal{C}\{\sigma\}$. Therefore, if $\{\nu\}$ is a $n$-tuple of normal states with $\{\nu\} \notin \mathcal{C}\{\sigma\}$ by a standard separation argument the existence of $\{x\} \subset \mathfrak{M}_{h}$ and $\alpha \in \mathbb{R}$ is implied such that

$$
\sum \nu_{k}\left(x_{k}\right)>\alpha \geq \sum \rho_{k}\left(x_{k}\right) \text { for all }\{\rho\} \in \mathcal{C}\{\sigma\} .
$$

Since all functionals involved are states, we might shift the $x_{k}$ into the positive cone of $\mathfrak{M}$. Hence, $x_{k} \geq \mathcal{O}$ and $\alpha \geq 0$ might be supposed from the very beginning. Especially, (5-2) implies

$$
\sup _{\left.T \in C P_{1}^{0(M)}\right)} \sum \nu_{k} \circ T\left(x_{k}\right)>\alpha \geq \sum \rho_{k}\left(x_{k}\right) \text { for all }\{\rho\} \subset C\{\sigma\}
$$

Note that the supremum on the left part of (5-3) has been taken over $C P_{1}^{0}(\mathfrak{M})$. Now, by the previously established equivalence $(3) \Leftrightarrow(4)$ and the definition (5-1) of $\mathcal{C}\{\sigma\}$ we may conclude that

$$
\sup _{\{\rho\} \subset \mathcal{C}\{\sigma\}} \sum \rho_{k}\left(x_{k}\right)=\sup _{T \in C P_{1}^{0}(\mathfrak{M})} \sum \sigma_{k} \circ T\left(x_{k}\right) .
$$

Let us take together (5-3) with (5-4). Then, we see that

$$
\{\nu\} \notin \mathcal{C}\{\sigma\} \Rightarrow K_{n}(\{\nu\},\{x\})>K_{n}(\{\sigma\},\{x\}) \text { for some }\{x\} \subset \mathfrak{M E}_{+} \text {. }
$$

On the other hand, if $\{\omega\} \in \mathcal{C}\{\sigma\}$, once more again argueing by $(3) \Leftrightarrow(4)$ we see the existence of $T \in C P_{1}^{0}(\mathfrak{M})$ with $\omega_{k}=\sigma_{k} \circ T, \forall k$. By definition of $K_{n}$ the latter implies

$$
K_{n}(\{\omega\},\{y\}) \leq K_{n}(\{\sigma\},\{y\}) \text { for all }\{y\} \subset \mathfrak{M}_{+} .
$$

Taking together (5-5) with (5-6) will yield

$$
\{\omega\} \in \mathcal{C}\{\sigma\} \Leftrightarrow K_{n}(\{\omega\},\{x\}) \leq K_{n}(\{\sigma\},\{x\}) \forall\{x\} \subset \mathbb{M} \mathcal{E}_{+} .
$$

Since $\{\omega\} \in \mathcal{C}\{\sigma\}$ is the same as (3), (5-7) means $(3) \Leftrightarrow(5)$. Hence, by the 
first part of the proof (5) is equivalent to each of the conditions (1)-(4).

\section{§6. A Special Result on AFD-Factors}

In this section we will suppose $\mathfrak{M}$ to be an AFD-factor over $\mathfrak{S}$. With the notations of Section 3. we then have:

Lemma 2. There exist a net $S_{\lambda}: \mathfrak{M} \rightarrow \mathfrak{U}_{0}$ of completely positive, $\sigma$-weakly continuous linear mappings of $\mathfrak{M}$ into $\mathfrak{N}_{0}$ such that

$$
K_{n}(\{\nu\},\{x\})=\lim _{\lambda} K_{n}\left(\{\nu\},\left\{S_{\lambda}(x)\right\}\right)
$$

for any choice of $\{x\} \subset \mathfrak{M C}_{+}$and each $n$-tuple $\{\nu\} \subset S_{0}(\mathfrak{M})$.

Proof. Assume $\varepsilon>0$, and $T \in C P_{1}^{0}(\mathfrak{M})$ chosen such that

$$
K_{n}(\{\nu\},\{x\})-\varepsilon \leq \sum \nu_{k} \circ T\left(x_{k}\right) .
$$

Because of [1, Theorem 6 (a) $\Leftrightarrow(d)]$ the factor $\mathfrak{M}$ is semidiscrete in the sense of [2]. Let $M_{m}$ denote the full algebra of $m \times m$-matrices. By [3] and semidiscreteness of $\mathfrak{M}$ there exist positive integers $\left(m_{\lambda}\right)_{\lambda \in \Lambda}$ and nets of $\sigma$-weakly continuous unital, completely positive linear maps $S_{\lambda}: \mathfrak{M} \rightarrow M_{m_{\lambda}}$ and $R_{\lambda}: M_{m_{\lambda}} \rightarrow \mathfrak{M} \mathcal{R}$ such that $R_{\lambda} \circ S_{\lambda}(x)$ converges $\sigma$-weakly to $x$ for all $x \in \mathfrak{M}$. Therefore, and with a view to $(6-2)$, there is $\lambda(\varepsilon)$ such that

$$
K_{n}(\{\nu\},\{x\})-2 \varepsilon \leq \sum \nu_{k} \circ T \circ R_{\lambda} \circ S_{\lambda}\left(x_{k}\right) \text { whenever } \lambda \geq \lambda(\varepsilon) .
$$

Let us associate, to each $\lambda \in \Lambda$, a natural number $N_{\lambda}$ such that $\operatorname{dim} \mathfrak{M}_{N_{\lambda}} \geq$ $m_{\lambda}$, and an orthoprojection $P_{\lambda} \in \mathfrak{M}_{N_{\lambda}}$ of (relative) dimension $m_{\lambda}$ in $\mathfrak{M R}_{N_{\lambda^{*}}}$ Then, $P_{\lambda} \mathfrak{M}_{N_{\lambda}} P_{\lambda}=F_{\lambda}$ is isomorphic to $M_{m_{\lambda}}$, and we instead might have supposed $S_{\lambda}$ : $\mathfrak{M} \rightarrow F_{\lambda}, R_{\lambda}: F_{\lambda} \rightarrow \mathfrak{M C}$ from the very beginning. Take this case, and let $\delta_{\lambda}$ be a normal conditional expectation from $\mathfrak{M}$ onto $\mathfrak{M}_{N_{\lambda}}$ (such a map exists, take the restriction of a normal conditional expectation of $\mathfrak{B}(\mathfrak{E})$ onto $\mathfrak{M}_{N_{\lambda}}$ to $\mathfrak{M}$ ). Then, $P_{\lambda} \delta_{\lambda}(\cdot) P_{\lambda}$ is a normal, unital, completely positive linear map of $\mathfrak{M}$ onto $F_{\lambda}$. Call this map $E_{\lambda}$. Then, $E_{\lambda}(x)=x$ for all $x \in F_{\lambda}$, and with $T_{\lambda}=R_{\lambda} \circ E_{\lambda}$ we have $R_{\lambda} \circ S_{\lambda}=R_{\lambda} \circ E_{\lambda} \circ S_{\lambda}=T_{\lambda} \circ S_{\lambda}$, with $T_{\lambda} \in C P_{1}^{0}(\mathfrak{M})$, which is due to normality of $E_{\lambda}$ and $\sigma$-weakly continuity of $R_{\lambda}$. Thus, by definition of $K_{n}$ :

$$
\sum \nu_{k} \circ T \circ R_{\lambda} \circ S_{\lambda}\left(x_{k}\right)=\sum \nu_{k} \circ T \circ T_{\lambda}\left(S_{\lambda}\left(x_{k}\right)\right) \leq K_{n}\left(\{\nu\},\left\{S_{\lambda}(x)\right\}\right) .
$$

On the other hand, with a fixed but arbitrarily chosen normal state $\mu$ we might construct maps $S_{\lambda}^{+} \in C P_{1}^{0}(\mathfrak{M})$ through the setting $S_{\lambda}^{+}(x)=S_{\lambda}(x)+\mu(x) P_{\lambda}^{\perp}$, $x \in \mathfrak{M}$. Since $\{x\} \subset \mathfrak{M q}_{+}$, we have $S_{\lambda}^{\perp}\left(x_{k}\right) \geq S_{\lambda}\left(x_{k}\right), \forall k$, and because all functionals 
involved are positive ones, we see $\sum \nu_{k} \circ T \circ S_{\lambda}\left(x_{k}\right) \leq \sum \nu_{k} \circ T \circ S_{\lambda}^{+}\left(x_{k}\right) \leq K_{n}(\{\nu\}$, $\{x\})$ for every $T \in C P_{1}^{0}(\mathfrak{M})$, hence

$$
K_{n}\left(\{\nu\},\left\{S_{\lambda}(x)\right\}\right) \leq K_{n}(\{\nu\},\{x\}) .
$$

Taking together (6-3), (6-4) and (6-5) yields $\left./ K_{n}(\{\nu\},\{x\})-K_{n}\left(\{\nu\},\left\{S_{\lambda}\right\}(x)\right\}\right) \mid$ $\leq 2 \varepsilon$ whenever $\lambda \geq \lambda(\varepsilon)$, from which the asserted relation (6-1) is deduced. By construction $S_{\wedge}(\mathfrak{M}) \subset F_{\lambda} \subset \mathfrak{M}_{N_{\lambda}} \subset \mathfrak{A}_{0}$ (cf. the definition of $\mathfrak{U}_{0}$ in Section 3).

\section{§7. An Application of a Structure Theorem for $\mathbb{C} . P_{0} . \mathbb{M}$ aps on Properly Imfinite $A F D$-Factors}

Adopting the notations of Sections 3 and 5 we have the following result in case of an properly infinite approximately finite dimensional factor $\mathfrak{M}$.

Lemma 3. For n-tuples $\{\omega\}$ and $\{\sigma\}$ of normal states over $\mathfrak{M}$ the following conditions are mutually equivalent:

$$
\begin{gathered}
K_{n}(\{\omega\},\{x\}) \leq K_{n}(\{\sigma\},\{x\}) \quad \text { for } \forall \cdot\{x\} \subset \mathfrak{U}_{0+} ; \\
\omega_{k / 2 \mathbb{2}}=\Phi\left(\sigma_{k / 2 \mathfrak{t}}\right), \forall k, \text { with } \Phi \in \mathcal{C}_{u}(\mathfrak{U}) .
\end{gathered}
$$

Proof. Since $\mathcal{C}_{u}(\mathfrak{U})$ is convex and point-1 $w^{*}$-compact in $\mathfrak{B}(\mathfrak{H} *)$ a standard separation argument applies and shows that (2) in the case of states is equivalent with

$$
\sup _{u} \sum \omega_{k}^{u}\left(x_{k}\right) \leq \sup _{u} \sum \sigma_{k}^{u}\left(x_{k}\right) \text { for all }\{x\} \subset \mathfrak{A}_{+}
$$

with the suprema extending over $\mathcal{U}(\mathfrak{H})$.

The map $\{x\} \rightarrow \sup \left\{\sum_{k} \omega_{k}^{u}\left(x_{k}\right): u \in \mathcal{U}(\mathfrak{U})\right\}=a(\{x\})$ is norm continuous on $n$-tuples of Hermitian elements. In fact, let $\{x\},\{y\}$ be such elements of $\mathfrak{A}$, $\varepsilon>0$, and $\left\|x_{k}-y_{k}\right\| \leq \varepsilon$, for all $k$. We may assume $a(\{x\}) \geq a(\{y\})$. Let $u \in \mathcal{U}(\mathfrak{A})$ with $\sum \omega_{k}^{n}\left(x_{k}\right) \geq a(\{x\})-\varepsilon$. Then, $-a(\{y\}) \leq-\sum \omega_{k}^{u}\left(y_{k}\right)$, and

$$
\begin{aligned}
0 & \leq a(\{x\})-a(\{y\}) \leq \varepsilon+\sum_{k}\left[\omega_{k}^{u}\left(x_{k}\right)-\omega_{k}^{u}\left(y_{k}\right)\right] \leq \varepsilon+\sum\left|\omega_{k}^{u}\left(x_{k}-y_{k}\right)\right| \\
& \leq \varepsilon+n\left\|x_{k}-y_{k}\right\| \leq(n+1) \varepsilon .
\end{aligned}
$$

Therefore, it is sufficient for (2) to hold that (7-1) proves valid only for all $n$-tuples $\{x\} \subset \mathfrak{A}_{+}$. (the latter set is uniformly dense in $\mathfrak{A}_{+}$).

The unitary group $\mathcal{U}(\mathfrak{U})$ of $\mathfrak{U}$ is $\sigma$-strongly* dense in the unitary group $\mathcal{U}(\mathfrak{M})$ of $\mathfrak{M}$ due to the fact that $\mathfrak{A}$ is generating for $\mathfrak{M}$ in the sense that $\mathfrak{U}^{\prime \prime}=\mathfrak{M}$, cf [14]. Therefore, for normal states $\{\nu\} \subset S_{0}(\mathfrak{M})$ we have 


$$
\sup _{u \in \mathcal{U}(\mathfrak{U})} \sum \nu_{k}^{u}\left(x_{k}\right)=\sup _{u \in \mathcal{U}(\mathfrak{M})} \sum \nu_{k}^{u}\left(x_{k}\right) \text { for } \forall\{x\} \subset \mathfrak{M} \mathbb{R}_{+},
$$

Now, $\mathfrak{M}$ has been supposed to be properly infinite. In such case it is a well-known fact that every isometry $v \in \mathfrak{M}$, i.e. $v^{*} v=1$, can be approximated strongly with unitary operators taken from $\mathfrak{M}$. Therefore, and since the linear forms involved are normal states, $\mathcal{U}(\mathfrak{M})$ in (7-2) might be replaced by the set of isometries over $\mathfrak{M}$ :

$$
\sup _{u \in \mathcal{U}(\mathfrak{M})} \sum \nu_{k}^{u}\left(x_{k}\right)=\sup \left\{\sum \nu_{k}\left(v^{*} x_{k} v\right): v^{*} v=1\right\}
$$

Assume we have a $n$-tuple $\{x\} \subset \mathfrak{A}_{0+}$. Then, there is $N$ such that $\{x\} \subset \mathfrak{M}_{N}$. Let $T \in C P_{1}^{0}(\mathfrak{M})$. Since $\mathfrak{M}$ is properly infinite a result of [5, Proposition 2.1] applies, saying that $v \in \mathfrak{M}$ exists such that $T(x)=v^{*} x v$, for all $x \in \mathfrak{M}_{N}$. Since $T$ is unital, $v$ is an isometry. On the other hand, every isometry $v \in \mathfrak{M}$ produces a completely positive, normal unital linear map $T: \mathfrak{M} \rightarrow \mathfrak{M}$ through the setting $T(x)=v^{*} x v$. Hence, our conclusion is

$$
\sup \left\{\sum \nu_{k}\left(v^{*} x_{k} v\right): v^{*} v=1\right\}=K_{n}(\{\nu\},\{x\})
$$

for every given $\{x\} \subset \mathfrak{A}_{0+}$ and all $\{\nu\} \subset S_{0}(\mathfrak{M} \mathcal{R})$.

Glueing together (7-1), (7-2), (7-3) with (7-4) and respecting the remark following (7-1) will give the desired equivalence $(1) \Leftrightarrow(2)$.

Remark. In conclusion, what we have proved is that

$$
K_{n}(\{\nu\},\{x\})=\sup _{u \in \mathcal{U}(\mathfrak{M})} \sum \nu_{k}^{u}\left(x_{k}\right)=\sup _{u \in \mathcal{U}(\mathfrak{R})} \sum \nu_{k}^{u}\left(x_{k}\right)
$$

for $\{\nu\} \subset S_{0}(\mathfrak{M})$ whenever the $n$-tuple $\{x\}$ belongs to $\mathfrak{A}_{+}$, provided $\mathfrak{M}$ is properly infinite. In case of a non-trivial $\mathrm{vN}$-algebra which is not properly infinite it is exactly (7-5) which is violated.

\section{§8. The Fimal Proof, Conclusions}

Let $\mathfrak{M}$ be a properly infinite AFD-factor over the separable, infinite dimensional Hilbert-space $\mathfrak{S}$. Assume two $n$-tuples $\{\omega\},\{\sigma\}$ of normal states over $\mathfrak{M}$ are given. Let $\Phi \in S T\left(\mathfrak{M}^{*}\right)$ with $\omega_{k}=\Phi\left(\sigma_{k}\right), \forall k$, i.e. (1) of our Theorem in Section 3 is supposed. This means (1) of Lemma 1. By Lemma 1, (1) is equivalent with (5), which implies (1) of Lemma 3 in a trivial manner. Due to Lemma 3 , (1) is equivalent with (2) of the formulation there. The latter, however, is the same as (2) of our Theorem, i.e. there is $\psi \in \mathcal{C}_{u}(\mathfrak{U})$ with $\omega_{k / 2)}=\psi\left(\sigma_{k / 2)}\right), \forall k$.

To see the other way around, suppose (2) of the Theorem is valid. Then, 
by Lemma 3

$$
K_{n}(\{\omega\},\{x\}) \leq K_{n}(\{\sigma\},\{x\}) \text { for all }\{x\} \subset \mathfrak{A}_{0+}
$$

has to hold. Let $\{y\} \in \mathfrak{M}_{+}$. Then, by (8-1) and Lemma 2

$$
K_{n}\left(\{\omega\},\left\{S_{\lambda}(y)\right\}\right) \leq K_{n}\left(\{\sigma\},\left\{S_{\lambda}(y)\right\}\right)
$$

for all $\lambda \in \mathcal{A}$ because of $S_{\lambda}\left(y_{k}\right) \in \mathfrak{Q}_{0+}, \forall k$. Taking the $\lambda$-limit of both sides in $(8-2)$ gives

$$
K_{n}(\{\omega\},\{y\}) \leq K_{n}(\{\sigma\},\{y\}),
$$

where we made use of $(6-1)$. Because $\{y\}$ could have been chosen arbitrarily in $\mathfrak{M E}_{+}$, condition (5) of Lemma 1 is satiesfied. By Lemma 1, the latter is equivalent with $\omega_{k}=\Phi\left(\sigma_{k}\right), \forall k$, with $\left.\Phi \in S T(M)^{*}\right)$. This completes the proof of the Theorem.

Remark. The only point where "properly infinite" really eniers is in proving (7-5). At this place, one could try to replace the unitary mixing condition by some other reasonable conditions in order to deal with the $n^{\text {* }}$ tuple problem for all AFD-factors (i.e. with the type $\mathbb{I}_{1}$ case included). Indeed, this is possible due to the first part of Proposition 2.1 of [5]. We omit the details and give only the result. The following conditions are equivalent:

$$
\begin{gathered}
\exists \bar{\Phi} \in S T\left(\mathfrak{D} \mathcal{R}^{*}\right) \text { with } \omega_{k}=\bar{\Phi}\left(\sigma_{k}\right), \forall k ; \\
\sup _{\left\{a_{j}\right\}} \sum_{j} \sum_{k} \omega_{k}\left(a_{j}^{*} x_{k} a_{j}\right) \leq \sup _{\left\{a_{j}\right\}} \sum_{j} \sum_{k} \sigma_{k}\left(a_{j}^{*} x_{k} a_{j}\right),
\end{gathered}
$$

for $\forall\{x\} \subset \mathfrak{Q}_{+}$, where the suprema have to be extended over all finite systenis of operators $\left\{a_{1}, \cdots, a_{m}\right\}$ in $\mathfrak{N}$ such that $\sum_{j} a_{j}^{*} a_{j} \leq 1$.

Let us consider now the hyperfinite $\mathbb{I I}_{1}$ factor. We will show explicitely that our Theorem cannot be true in this case (cf. the remark at the end of Section 7).

We shall take the simplest possible case: $n=1$. Take the canonical trace $\tau$ as one of the states. Assume $\omega \neq \tau$ is a state of $S_{0}(\mathfrak{M})$. Since $\tau$ is unitarily invariant, $\omega \notin\left\{\mu: \mu_{/ \mathfrak{X}}=\Phi(\tau, \mathfrak{l}), \Phi \in \mathcal{C}_{u}(\mathfrak{U})\right\} \equiv\{\tau\}$, trivially. On the other hand, the map $T(x)=\omega(x) 1$ is in $C P_{1}(\mathfrak{M})$, and $\tau \circ T=\omega$, which shows the violation of the assertion of our Theorem in the finite case.

\section{§9. The m-Tuple-Problem on Other Algeliras}

Let $\mathfrak{M}$ be a vN-algebra over $\mathfrak{S}$ which is compatible to a properly infinite 
AFD-factor $\mathfrak{R}$ in the sense that $\mathfrak{M} \subset \mathfrak{R}$ and a normal conditional expectation $E$ of $\mathfrak{N}$ onto $\mathfrak{M}$ exists.

Assume $\mathfrak{U}$ is a generating U.H.F.-subalgebra of $\mathfrak{R}$, i.e. $\quad \mathfrak{U}^{\prime \prime}=\mathfrak{N}$ and $\mathfrak{A}=$ $\overline{\cup \mathfrak{N}_{N}}$, with an increasing sequence of finite dimensional subfactors. Then, we may use the information of our Theorem to find conditions for the $n$-tuple problem over the compatible subalgebra $\mathfrak{M}$.

Proposition. Let $\{\sigma\},\{\omega\} \subset S_{0}(\mathfrak{M})$ be two n-tuples of normal states over M. The subsequently listed assertions are equivalent:

$$
\begin{gathered}
\exists \Phi \in S T\left(\mathfrak{M}^{*}\right) \text { with } \omega_{k}=\Phi\left(\sigma_{k}\right), \forall k ; \\
\exists \psi \in \mathcal{C}_{u}(\mathfrak{U}) \text { such that } \omega_{k} \circ E_{\text {/2X }}=\psi\left(\sigma_{k} \circ E_{/ \mathfrak{Q}}\right), \forall k .
\end{gathered}
$$

Proof. Suppose (1). By Lemma 1 (which is true on a general $\mathrm{vN}$-algebra), there is $T \in C P_{1}^{0}(\mathfrak{M})$ with $\omega_{k}=\sigma_{k} \circ T, \forall k$. Therefore, $\omega_{k} \circ E=\left(\sigma_{k} \circ E\right) \circ T \circ E$ since $E \circ T=T$ ( $E$ projects onto $\mathfrak{M})$, and $T \circ E \in C P_{1}^{0}(\mathfrak{N})$, for both $T$ and $E$ are normal, completely positive and unital, acting over $\mathfrak{M}$, resp. from $\mathfrak{N}$ onto $\mathfrak{M}$. Hence, our Theorem applies with respect to the $n$-tuples of normal states $\{\omega \circ E\}$ and $\{\sigma \circ E\}$ over the properly infinite AFD-factor $\mathfrak{R}$, and (2) follows.

To see the other direction, suppose (2) holds. By our Theorem, $\exists \psi \in S T\left(\Re^{*}\right)$ such that $\omega_{k} \circ E=\psi\left(\sigma_{k} \circ E\right), \forall k$. By Lemma 1 we get $T \in C P_{1}^{0}(\mathfrak{N})$ such that $\omega_{k} \circ E=\sigma_{k} \circ E \circ T, \forall k$. The mapping $S=E \circ T_{/ M}$ is normal, unital and completely positive over $\mathfrak{M}$. Since $E$ projects onto $\mathfrak{M}$ we see $\omega_{k}=\sigma_{k} \circ S$, for all $k$. By Lemma 1 the assertion (1) follows.

Remark. By the just proved all cases of the $n$-tuple-problem on arbitrary AFD-factors over separable $\mathfrak{S}$ are covered and seen from a common point of view, including the finite type factors and the finite dimensional factor case (take $\mathfrak{N}=\mathfrak{M} \bar{\otimes} \mathfrak{B}\left(l^{2}\right)$ in the Proposition).

Moreover, one can deduce all known conditions for the commutative case (which has been treated in [7] extensively) in a very comprehensive way from our Proposition when $\mathfrak{M}$ is seen as a compatible subalgebra* of a suitably chosen properly infinite AFD-factor $\mathfrak{l}$ over $\mathfrak{S}$. Again, for the special case $\mathfrak{B}(\mathfrak{E})$ this problem has been considered in [10] ( $\mathfrak{M}$ can be every atomic type subalgebra of $\mathfrak{N}=\mathfrak{B}(\mathfrak{S}))$.

In [12] an attempt was made to base the $n$-tuple-problem heavily on the commutative case provided a condition of "almost commutativity" for the $n$ tuples of states considered holds. This case now also follows at once from the

\footnotetext{
* We refer to the terminology of [13].
} 
Proposition above (besides the fact that the problems setting in [12] is in the slightly more general frame of $C^{*}$-algebras). But note that in [6]-[10], [12] all formulations read in terms of $S T\left(\mathfrak{M}^{*}\right)$, which seem rather "esoteric" to work with, whereas now we have the useful Lemma 1 to reduce the $n$-tuple problem between normal states to a problem about normal maps in any case (in [7] a very special form of the equivalences (1)-(4) of Lemma 1 has been derived for some abelian vN-algebra). This fact can now be expressed in a very useful representation theorem for c.p.-stochastic maps over $\mathfrak{M}_{*}$ through unitary mixing maps over a generating U.H.F.-C*-subalgebra.

\section{§10. A Representation Theorem for $\mathbb{C}_{0} \mathbb{P}_{\text {o-Stochastic }} \mathbb{M}$ Mps}

Over the Predual of a Non-Finite $\mathbb{A F D}$-Factor

With the notations of Sections 1 and 3 , let $j_{2 \mathfrak{}}$ be defined as the bijection $j_{2 \mathfrak{X}}$ : $\mathfrak{M}_{*} \rightarrow \mathscr{A}_{*}$, with $\omega \mapsto \omega_{/ \mathfrak{2}}$, where $\mathfrak{P}_{*}$ is the set of all bounded linear forms over $\mathfrak{A}$ which arise as restrictions of $\sigma$-weakly continuous linear forms on $\mathfrak{M}$ onto $\mathfrak{A}$. Let us consider a subset of $\mathcal{C}_{u}(\mathfrak{U})$, given by $\mathcal{C}_{u}^{0}(\mathfrak{P})=\left\{\Phi \in \mathcal{C}_{u}(\mathfrak{U}): \Phi\left(\mathfrak{P}_{*}\right) \subset \mathfrak{A}_{*}\right\}$. All we have proved can now be reformulated into the following result.

Corollary. Let $\mathfrak{M}$ be a non-finite AFD-factor. Then

$$
S T\left(\mathfrak{M}_{*}\right)=j_{\mathfrak{X}}^{-1} \circ \mathcal{C}_{u}^{0}(\mathfrak{U}) \circ j_{\mathfrak{A}},
$$

i.e. to every completely positive, siate-preserving linear map $\psi$ acting over the predual of $\mathfrak{M}$ there exists a unitary mixing map̄ping $\Phi$ ovev $\mathfrak{U} *$, which eaves $\mathfrak{U}_{*}$ giobally invariant, such that $j_{\mathfrak{2}} \circ \psi=\Phi \circ j_{\mathfrak{2}}$.

Proof. That each element of the right hand set of (10-1) belongs to $S T\left(\mathfrak{M}_{*}\right)$ follows since all mappings involved are completely positive as well as statepreservingly acting. To see the other way around, let $\psi \in S T\left(\mathfrak{M R}_{*}\right)$. By $\{\mathfrak{\Im}, \geq\}$ let us denote the increasingly directed (in the sense of inclusion of sets) family of finite subsets of $S_{0}(\mathfrak{M})$. By our Theorem, to each set of $\mathfrak{\Im}$, say $G \in \mathfrak{F}$, there is a $\Phi_{G} \in \mathcal{C}_{u}(\mathfrak{U})$ such that

$$
\psi(\omega)_{, 2 t}=\Phi_{G}\left(\omega_{\text {קथी }}\right), \forall \omega \in G .
$$

Knowing the $\mathcal{C}_{u}(\mathfrak{U})$-set being point- $w^{*}$-compact assures the existence of a converging subnet $\left\{\Phi_{G_{\lambda}}\right\}$ of $\left\{\Phi_{G}\right\}$, with

$$
\text { point- } w^{*}-\lim _{\lambda} \Phi_{G_{\lambda}}=\Phi \in \mathcal{C}_{u}(\mathfrak{Z}) \text {. }
$$

Let $\omega \in S_{0}(\mathfrak{M})$. Then, there is $\lambda(\omega)$ such that for $\lambda \geq \lambda(\omega) \omega \in G_{\lambda}$. Therefore, 
by $(10-2) \psi(\omega)_{/ \Re}=\Phi_{G_{\lambda}}\left(\omega_{/ 2 \lambda}\right), \forall \lambda \geq \lambda(\omega)$, which relation implies by means of $(10-3)$ that

$$
\psi(\omega)_{/ 2 \lambda}=\lim _{\lambda} \Phi_{G_{\lambda}}\left(\omega_{\text {/2X }}\right)=\Phi\left(\omega_{\text {/2X }}\right) .
$$

Since $\psi \in S T\left(M_{*}\right)$, i.e. $\psi$ maps the predual into itself, and (10-4) holds for each $\omega \in S_{0}(\mathfrak{M})$, we see both $\Phi\left(\mathfrak{U}_{*}\right) \subset \mathfrak{U}_{*}$ and $j_{\mathfrak{N}} \circ \psi=\Phi \circ j_{\mathfrak{V}}$ with a $\Phi \in \mathcal{C}_{u}(\mathfrak{U})$, and the proof is complete.

Remark. With view to our Proposition and the remark in Section 9 it should be clear how to modify the assertion of the Corollary both for the finite dimensional factor and the hyperfinite factor case and all thoer non-factorial cases which can be considered to be compatible with one of the non-finite AFD-factors we dealt with.

\section{Acknowledgements}

In part the present research was done and finished while the author was visiting the ETH Zurich in winter of $1985 / 86$. I wish to thank Prof. $\mathbb{H}$. Primas and his collaborators of the Laboratorium fur Physikalische Chemie of the ETH for their kind hospitality and many fruitful discussions. Also I am gratefu] for many exiting discussions with Prof. Dr. A. Uhlmann, Sektion Physik der KMU Leipzig, on related problems in an early stage of this work.

\section{References}

[1] Connes, A., Classification of injective factors, Ann. Math. 104 (1976), 73-115.

[2] Effros, E.G. and Lance, C., Tensor products of operator algebras, Adv. Math. 25 (1977), 1-34.

[3] Choi, M.-D. and Effros, E.G., Nuclear $C^{*}$-algebras and the approximation property, Am. J. Math. 100 (1978), 61-79.

[4] Elliott, G.A. and Woods, E.J., The equivalence of various definitions for a properly infinite vN-algebra to be AFD, Proc. AMS 60 (1976), 175-179.

[5] Haagerup, U., A New Proof of the Equivalence of Injectivity and Hyperfiniteness for Factors on a Separable Hilbert Space, Journ. Funct. Analysis 62, (1985), 160-200.

[6] Alberti, P.M. and Uhlmann, A., Stochasticity and Partial Order (Doubly Stochastic Maps and Unitary Mixing), D. Reidel Publ. Comp., Dordrecht, 1982.

[7] - Dissipative Motion in State Spaces, Teubner-Texte zur Mathematik, Bd.33, Teubner-Verlag, Leipzig, 1981.

[8] Alberti, P.M., On the simultaneous transformation of density operators by means of a c.p., unity preserving linear map, Publ. RIMS Kyoto Univ. $2 \mathbb{1}$ (1985), 619-644.

[9] Uhlmann, A., The transition probability for states of *algebras, ZiF-Preprint No. 62, Bielefeld, 1984.

[10] Alberti, P.M., Vollständig positive Abbildungen und verallgemeinerte Ubergangswahrscheinlichkeiten über atomaren vN-Algebren, Wiss. Z. KMU, MNR. 34, H.6, (1985), 
572-579.

[11] Sakai, S., $C^{*}$-algebras and $W^{*}$-algebras, Springer-Verlag, Berlin-Heidelberg-New York, 1971.

[12] Uhlmann, A., On stochastic maps of $\mathbb{C}^{*}$-algebraic state spaces, in: Ergodic Theory and Related Topics, Vol. 12 of Mathematical Research, Akademieverlag, Berlin, 1982, p. 185-195.

[13] Arveson, W.B., Analyticity in Operator Algebras, Am. J. Math. 89 (1967), 578-642.

[14] Glimm, J., Kadison, R.V., Unitary operators in C*-algebras, Pacific J. Math. 10 (1960), $547-556$. 
